\title{
An intensive swallowing exercise protocol for improving swallowing physiology in older adults with radiographically confirmed dysphagia
}

This article was published in the following Dove Medical Press journal: Clinical Interventions in Aging

\author{
Matina Balou' \\ Erica G Herzberg ${ }^{2}$ \\ David Kamelhar ${ }^{3}$ \\ Sonja M Molfenter ${ }^{4}$ \\ 'Department of Otolaryngology, \\ New York University School of \\ Medicine, Head \& Neck Surgery, \\ New York, NY, USA; ${ }^{2}$ Department \\ of Speech-Language Pathology, New \\ York University Langone Health, \\ Rusk Rehabilitation, New York, \\ NY, USA; ${ }^{3}$ New York University \\ School of Medicine, Department \\ of Pulmonary Medicine, New \\ York, NY, USA; ${ }^{4}$ Department of \\ Communicative Sciences and \\ Disorder, Communicative Sciences \\ and Disorders, New York University \\ Steinhardt, New York, NY, USA
}

Correspondence: Sonja M Molfenter Communicative Sciences and Disorders, New York University Steinhardt, 665 Broadway, Room 928, New York, NY 10012 , USA

Tel +l 2129927694

Emailsmm16@nyu.edu
Purpose: The aim of this study was to investigate improvements in swallowing function and physiology in a series of healthy older adults with radiographically confirmed dysphagia, following completion of an exercise-based swallowing intervention.

Patients and methods: Nine otherwise healthy older adults (six females, mean age $=75.3$, $\mathrm{SD}=5.3$ ) had confirmed impairments in swallowing safety and/or efficiency on a modified barium swallow study. Each participant completed an 8-week swallowing treatment protocol including effortful swallows, Mendelsohn maneuvers, tongue-hold swallows, supraglottic swallows, Shaker exercises and effortful pitch glides. Treatment sessions were conducted once per week with additional daily home practice. Penetration-Aspiration Scale and the Modified Barium Swallowing Impairment Profile (MBSImP) were scored in a blind and randomized fashion to examine changes to swallowing function and physiology from baseline to post-treatment.

Results: There were significant improvements in swallowing physiology as represented by improved oral and pharyngeal composite scores of the MBSImP. Specific components to demonstrate statistical improvement included initiation of the pharyngeal swallow, laryngeal elevation and pharyngeal residue. There was a nonsignificant reduction in median PAS scores. Conclusion: Swallowing physiology can be improved using this standardized high-intensity exercise protocol in healthy adults with evidence of dysphagia. Future research is needed to examine the individual potential of each exercise in isolation and to determine ideal dose and frequency. Studies on various etiological groups are warranted.

Keywords: dysphagia, modified barium swallow, rehabilitation, deglutition, exercise, presbyphagia

\section{Introduction}

Current evidence suggests that $15 \%$ of people over 65 years old experience dysphagia (a disruption to swallowing function). ${ }^{1}$ By extension, a staggering 235 million older adults will suffer from a swallowing disorder worldwide in $2050 .^{2}$ While the act of swallowing food and liquid is a basic human function that most people take for granted, safe and efficient swallowing is actually an extremely complex neuromuscular process. The pharynx must rapidly (temporarily) reorganize from its primary breathing configuration to a swallowing configuration through the timely sequential contraction and relaxation of many muscles to allow a bolus (mass of food/liquid) to pass safely from the mouth to the esophagus without entering the airway. ${ }^{3,4}$ Failure in these mechanisms can result in an "unsafe" swallow (bolus enters the laryngeal vestibule and is at risk of being aspirated into the lungs) and/or an "inefficient" swallow (unswallowed bolus, known as "residue", remains in the pharynx after swallowing). The gold-standard method for assessing swallowing safety and efficiency is through dynamic imaging 
of radiopaque boluses (usually barium) swallowed during a modified barium swallow study (MBSS). Impaired swallowing has been associated with negative health sequelae including nutrition/hydration consequences, aspiration pneumonia and significant reductions in quality of life..$^{5-10}$

Age-related atrophy (sarcopenia) of the swallowing musculature has been widely adopted as an explanation for age-related decline in swallow function. Sarcopenia has been established in the tongue, ${ }^{11-14}$ geniohyoid muscle ${ }^{15}$ and the pharyngeal constrictors. ${ }^{16,17}$ It follows then, that exercises that target the swallowing muscles are desirable for reversing sarcopenia and age-related dysphagia. Yet, despite being widely adopted in clinical practice, the benefit of most swallowing exercises to rehabilitate the swallowing muscles is poorly understood. ${ }^{18}$

The opportunity to contribute to this gap in the literature arose in the context of recruiting a sample of healthy older control subjects for an unrelated study. Nearly 100 patients served as controls and during the process of completing their MBS studies, nine individuals over 65 years old were observed to have evidence of dysphagia (impaired safety and/or efficiency). These otherwise healthy older adults were offered the opportunity to complete an intense 8-week swallowing exercise protocol. Our hypothesis was that an intense 8-week swallowing exercise protocol would result in improvements in swallowing physiology manifesting in improved swallowing safety and efficiency.

\section{Methods}

\section{Participants}

This study was approved by the NYU School of Medicine Institutional Review Board (IRB) (S1201987). Nine healthy older adults (six females) were included in this retrospective analysis ranging from 67 to 86 years old (mean $=75.3$, $\mathrm{SD}=5.3$ ). Specific inclusion criteria were any single swallow with a safety impairment (Penetration-Aspiration Scale $[\mathrm{PAS}]^{19}$ score of 3 or higher) and/or any single swallow with an efficiency impairment (Modified Barium Swallow Impairment Profile $[\mathrm{MBSImP}]^{20}$ Component 16 Pharyngeal Residue score of 2 or higher). These criteria for initiating treatment follow standard clinical procedures at this institution and thus IRB-approved waiver for consent to participate was obtained.

\section{Modified Barium Swallow Study}

Each participant completed a standardized MBSS before and after the treatment protocol. Continuous fluoroscopy was delivered using a Siemens fluoroscope (Iconos R200) and captured at a rate of 30 frames per second on a Digital
Swallow Workstation (Kay Pentax) or TIMS (TIMS Medical), given that the facility switched capture systems during the course of this study. Each participant swallowed two Varibar (Bracco Imaging) barium boluses for each of the following conditions: $1 \mathrm{~mL}$ thin liquid, $3 \mathrm{~mL}$ thin liquid, $5 \mathrm{~mL}$ thin liquid, $10 \mathrm{~mL}$ thin liquid, uncalibrated cup sip thin liquid, continuous thin liquid drinking and $5 \mathrm{~mL}$ pudding. The final bolus in the protocol was one-quarter of hard solid (cracker) coated with Varibar pudding. Participants self-fed and all liquids were delivered by medicine cup. At the discretion of the assessing clinician, protocol deviations were made online to maximize patient safety and yield of clinical information. It should be noted that this MBSS protocol deviates slightly from the MBSImP recommended protocol; however, it does include a wide range of bolus volumes and consistencies which is known to be important in eliciting impairment in MBSImP scores. ${ }^{21}$

\section{Swallowing exercises}

Each eligible participant was scheduled for 8 weeks of treatment within 2 weeks of the initial MBSS completion. Treatment sessions were conducted once per week in a hospital-based outpatient clinic and lasted for 45 minutes. The swallowing exercises in this study included effortful swallows, tongue-hold swallows (also known as the Masako maneuver), supraglottic swallows, Shaker exercises, Mendelsohn maneuvers and finally effortful pitch glides. Table 1 outlines the underlying target for each exercise and the instructions used to teach each exercise. The targeted number of repetitions for each set of exercises was 20 , except the effortful pitch glide where the target was 10 per set (total swallow exercises per treatment session $=110$ ). If a participant could not tolerate 20 repetitions of a particular exercise in the beginning, a modified incremental increase was executed. By the end of the 8 weeks, all participants could tolerate completion of the targeted repetitions per set. In addition to weekly treatment sessions, participants were expected to complete three additional sets of daily homework for a total of 330 swallow repetitions per day. This regimen of exercises at this frequency is standard of care for patients with evidence of impaired swallowing safety and efficiency at this outpatient clinic. These exercises were applied to all participants regardless of their underlying swallowing physiology. This regimen was designed with several important factors in mind. First, it balances a mix of both swallowing and non-swallowing exercises and therefore draws on the neuroplasticity principles of both specificity and transference. ${ }^{22,23}$ Furthermore, the number of repetitions 
Table I Swallowing exercises completed in each set of exercises

\begin{tabular}{|c|c|c|c|}
\hline Exercise & $\begin{array}{l}\text { Reps per } \\
\text { set }\end{array}$ & Targeted outcome & Instruction \\
\hline Effortful swallow & 20 & $\begin{array}{l}\text { Increase muscular activation of the pharyngeal } \\
\text { constrictors and base of tongue }{ }^{25}\end{array}$ & $\begin{array}{l}\text { I. Push your tongue up to the roof of your mouth } \\
\text { 2. Swallow "hard" with effort } \\
\text { 3. Pretend you are swallowing a golf ball }\end{array}$ \\
\hline Tongue-hold swallow & 20 & $\begin{array}{l}\text { Increased contraction of the superior pharyngeal } \\
\text { constrictor muscle }\end{array}$ & $\begin{array}{l}\text { I. Hold your tongue gently between your teeth } \\
\text { 2. Swallow your saliva with your tongue in this } \\
\text { position }\end{array}$ \\
\hline Supraglottic swallow & 20 & Volitional laryngeal vestibule closure ${ }^{27}$ & $\begin{array}{l}\text { I. Take a breath and hold it } \\
\text { 2. Swallow with effort } \\
\text { 3. Cough }\end{array}$ \\
\hline Shaker exercise & 20 & Strengthening of hyolaryngeal elevation muscles ${ }^{28}$ & $\begin{array}{l}\text { I. Lie flat on your back } \\
\text { 2. Lift only your head and look at your toes } \\
\text { (do not lift your shoulders) } \\
\text { 3. Hold this position for I second and then lower } \\
\text { your head }\end{array}$ \\
\hline Mendelsohn maneuver & 20 & $\begin{array}{l}\text { Volitional prolonging of hyolaryngeal elevation and } \\
\text { upper esophageal sphincter opening }{ }^{29}\end{array}$ & $\begin{array}{l}\text { I. Begin a regular swallow } \\
\text { 2. As you feel your larynx move in an upward } \\
\text { direction, squeeze your muscles } \\
\text { 3. Maintain this position for up to } 5 \text { seconds } \\
\text { 4. Relax and finish the swallow }\end{array}$ \\
\hline Effortful pitch glide & 10 & Shortening and constriction of the pharyn $x^{30}$ & $\begin{array}{l}\text { I. Take a deep breath } \\
\text { 2. Say the sound "eee" with effort moving from a } \\
\text { low pitch to a high pitch }\end{array}$ \\
\hline
\end{tabular}

is high with the principle of intensity in mind. Finally, the duration of the protocol was selected to extend beyond the minimum of 5 weeks of exercise which are recommended to demonstrate improvements in skeletal muscle function. ${ }^{24}$ This study provided an opportunity to evaluate the effectiveness of the dysphagia rehabilitation protocol at our institution in a series of individuals not prone to spontaneous recovery or disease progression. Unfortunately, adherence to homework was not tracked in this retrospective analysis.

\section{Data analysis}

Each MBSS was scored for the PAS and the MBSImP by experienced MBSImP certified clinicians who were blind to the time point (pre- vs post-treatment). The PAS is an 8-point ordinal rating scale that reflects the depth of material entering the airway and the body's response to that invasion. ${ }^{19}$ Scores of 1 and 2 are considered normal, 3-5 represent penetration and 6-8 represent aspiration. The MBSImP is a standardized rating system to quantify 17 components of swallowing physiology. ${ }^{31}$ Each component has operationalized definitions for scoring on an ordinal scale. MBSImP scores were completed according to the standard "overall impression" scoring protocol where the worst score for a given component is assigned across all bolus volumes and consistencies. Note that component 1 (lip closure) could not be rated in five of the studies due to fluoroscopy collimation and therefore it was dropped from this analysis. Remaining oral components were summed (components 2-6) to create the "oral total" (OT) score and pharyngeal components were summed (components 7-16) to create the "pharyngeal total" (PT) score. The esophageal phase (component 17) was not included in this analysis as it was not the target of these rehabilitative exercises. Finally, the worst PAS score across the entire study was recorded. Reliability of the PAS was established on $20 \%$ of the larger dataset $(n=163$, intrarater: $\mathrm{ICC}=0.92,95 \% \mathrm{CI}=0.85-0.97$; interrater: $\mathrm{ICC}=0.92$, $95 \% \mathrm{CI}=0.84-0.97)$. MBSImP scores were deemed reliable through the rigorous certification standards of $80 \%$ agreement across each component during training (for more information, see https://www.mbsimp.com/).

\section{Statistical analysis}

To analyze the overall within-subject change in swallowing function from pre-treatment to post-treatment, Wilcoxon Signed-Rank Tests were run on MBSImP OT, MBSImP PT and PAS scores. In addition, McNemar's test was used to compare binary PAS scores of safe (PAS 1-2) vs unsafe (PAS 3-8) by treatment time point. Where an overall MBSImP score (OT or PT) was significantly improved, further posthoc Wilcoxon Signed-Rank Tests explored in which specific 
MBSImP components had significantly changed. Two-tailed $P$-values $<0.05$ were considered statistically significant.

\section{Results}

At baseline, all nine participants had impaired swallowing safety and/or efficiency (per the inclusion criteria). Specifically, three participants demonstrated impaired swallowing safety only (PAS score of 3 or higher), two participants demonstrated impaired efficiency only (MBSImP Component 16 Pharyngeal Residue score 2 or higher) and the remaining four participants had both safety and efficiency concerns.

A summary of the statistical analysis appears in Table 2. Analysis of PAS scores indicated that the swallowing safety did not improve significantly post-treatment, despite the median score improving from a 3 (unsafe/penetration) to 1 (safe/normal) post-treatment. Given that there are other methods to analyzing the PAS data ${ }^{32}$ we ran a second test to confirm our finding. The result was unchanged when we categorized the PAS data in a binary fashion ( 1 and 2 vs 3 through 8) [McNemar, $P=0.125]$. However, the analysis of MBSImP scores showed significant reductions (improvements) in both OT scores and in PT scores.

Analysis of individual MBSImP components revealed that three components demonstrated significant differences from pre- to post-treatment scores. Component 6, initiation of the pharyngeal swallow, improved from pre-treatment (median $=3$, range $1-3)$ to post-treatment (median $=1$, range $1-3)[Z=-2.1, P=0.038]$. Component 8 , laryngeal elevation, also significantly improved from pre-treatment (median $=1$, range $0-1$ ) to post-treatment (median $=0$, range $=0-1)[Z=-2.2$, $P=0.025]$. And Component 16, pharyngeal residue, improved from pre-treatment (median $=2$, range $1-2$ ) to post-treatment ( median $=1$, range $=0-2$ ) $[\mathrm{Z}=-2.0, P=0.046]$.

\section{Discussion}

A significant proportion of healthy older adults experience negative changes to swallowing function as the result of aging. ${ }^{9,33-37}$ Exploring methods to prevent and/or reverse these changes is critical for our rapidly aging society especially given the known health and quality of life disruptions associated with dysphagia. Unfortunately, the rehabilitative potential of most swallowing exercises is poorly understood, despite being widely adopted in clinical practice.

This study was performed in a cohort of healthy older adults with radiographically confirmed dysphagia. Healthy individuals rarely receive instrumental assessments to confirm dysphagia and thus the opportunity to test intervention in symptomatic but healthy older adults provides a novel contribution to the literature. Our results indicated that there was no significant change in swallowing safety through our analysis of pre- vs post-treatment PAS scores. This small sample was almost certainly underpowered. Yet, it is worth noting that the descriptive data show a reduction in median PAS score over the course of treatment (from 3 to 1). This is a clinically meaningful shift from a deviant score (penetration) to a normal score (no airway invasion). Of course, replication with statistical significance in larger samples must be conducted before a conclusive statement regarding swallowing safety can be made.

Promisingly, we found that this exercise protocol improved blinded ratings of swallowing physiology captured using the MBSImP - a standardized rating method for 17 physiological components of swallowing. Statistically significant reductions (ie, improvements) in overall OT and PT scores were observed. Specific components that had statistical improvements included initiation of the pharyngeal swallow, laryngeal elevation and pharyngeal residue. It should be noted that initiation of the pharyngeal swallow captures the depth of the bolus at the moment of onset of brisk anterior-superior hyoid movement and is known to vary in healthy populations. ${ }^{38,39}$ Improvements in this component may reduce risk of swallowing safety concerns; however, high scores on this component alone should not define dysphagia.

Table 2 Comparison of swallowing impairment and physiology from pre-treatment to post-treatment

\begin{tabular}{|l|l|l|l|l|l|l|}
\hline & \multicolumn{2}{|l|}{ PAS score } & \multicolumn{2}{l|}{ MBSImP oral total } & \multicolumn{2}{l|}{ MBSImP pharyngeal total } \\
\cline { 2 - 7 } & Pre-tx & Post-tx & Pre-tx & Post-tx & Pre-tx & Post-tx \\
\hline Median & 3 & $\mathrm{I}$ & 4 & 4 & 10 & 7 \\
\hline Min & $\mathrm{I}$ & $\mathrm{I}$ & 2 & $\mathrm{I}$ & 7 & 3 \\
\hline Max & 7 & 8 & 9 & 6 & 14 & 11 \\
\hline$Z$ & -0.42 & 0.67 & -2.132 & & -2.257 \\
\hline P & & $\mathbf{0 . 0 3 3}$ & $\mathbf{0 . 0 2 4}$ \\
\hline
\end{tabular}

Note: Bolded results signify statistical significance.

Abbreviations: Max, maximum; Min, minimum; MBSImP, Modified Barium Swallowing Impairment Profile; PAS, Penetration-Aspiration Scale; Tx, treatment. 
Some of the exercises used in our protocol have been studied for their immediate impact on swallowing but not as rehabilitative exercises for individuals with dysphagia. For example, recent research using simultaneous pharyngeal manometry and intramuscular electromyography confirms that the tongue-hold maneuver significantly increases the activation of the superior pharyngeal constrictor, as well as the genioglossus and the submental musculature. ${ }^{26}$ Muscle function MRI data reveal that the effortful pitch glide elicits greater muscle activation than swallowing ${ }^{40}$ and subsequent data collected using dynamic MRI revealed that the effortful pitch glide had equivalent motor output to swallowing. ${ }^{30}$ However, to the best of our knowledge, this is the first rehabilitation investigation using these exercises in individuals with confirmed swallowing impairments.

While our study design does not allow us to empirically test the relationship between individual exercises and physiological outcomes, the significant results do align with plausible theoretically informed relationships. For example, the improvement in bolus location at swallow onset may be related to training volitional airway closure during the supraglottic swallow maneuver. Improvements in pharyngeal residue may be the result of improved strength in the pharyngeal constrictor muscles through training of effortful and tongue-hold swallows and/or by prolonged upper esophageal sphincter opening during Mendelsohn maneuvers. Finally, improvements in laryngeal elevation could be linked to improved shortening induced by the effortful pitch glide, the strengthening of the hyolaryngeal muscles in the Shaker exercise and/or to the volitional prolongation of laryngeal elevation during the Mendelsohn maneuver.

This study is not without limitations. First, this case-series lacked a control group. Furthermore, the interventions applied in this study did not specifically target each individual's underlying deficits in their swallowing physiology, rather the intervention protocol was a predetermined set of swallowing and non-swallowing exercises that are widely adopted in clinical practice. Related to this, we did not test any exercise in isolation and therefore, cannot determine which of the exercises provided the greatest rehabilitative potential for this sample. Finally, we did not track participant adherence with homework exercises and therefore do not know how many repetitions of exercises each participant completed. We recognize that future work must address these issues.

\section{Conclusion}

Older adults are vulnerable to developing dysphagia, both through increased prevalence of medical conditions associated with dysphagia (ie, stroke, progressive neurological conditions, head and neck cancer) and through changes associated with aging (ie, sarcopenia). Yet, there is a dearth of literature examining the effectiveness of swallowing exercises to rehabilitate swallowing function. This study provided an opportunity to test the impact of an 8-week treatment regimen comprised of widely adopted (yet poorly understood) swallowing exercises in a sample of healthy older adults with documented dysphagia but not prone to spontaneous recovery or disease progression. Our results indicated that the treatment regimen completed by these individuals was associated with positive changes to swallowing physiology including improvements in swallow initiation, laryngeal elevation and postswallow residue. Future prospective research investigating the rehabilitative potential of each individual exercise is needed.

\section{Acknowledgments}

The authors would like to acknowledge Giovanna Castillo for her assistance with swallow study preparation and organization. This work was supported by funding from Lerner, NTM Info \& Research and Wolstencroft Funds. A portion of this research has been accepted for presentation at the 2019 Dysphagia Research Society meeting.

\section{Disclosure}

SMM receives grant funding from the NIH NIDCD R21DC015067. MB and DK receive salary from NYU School of Medicine, EH from NYU Rusk and SMM from NYU Steinhardt. The authors report no other conflicts of interest in this work.

\section{References}

1. Madhavan A, Lagorio LA, Crary MA, Dahl WJ, Carnaby GD. Prevalence of and risk factors for dysphagia in the community Dwelling elderly: a systematic review. J Nutr Health Aging. 2016;20(8):806-815.

2. He W, Goodkind D, Kowal P. International Population Reports: An Aging World 2015. International population reports, P95/16-1. US Census Bureau; 2016.

3. Jean A. Brain stem control of swallowing: neuronal network and cellular mechanisms. Physiol Rev. 2001;81(2):929-969.

4. Ertekin C, Aydogdu I. Neurophysiology of swallowing. Clin Neurophysiol. 2003;114(12):2226-2244.

5. Ekberg O, Hamdy S, Woisard V, Wuttge-Hannig A, Ortega P. Social and psychological burden of dysphagia: its impact on diagnosis and treatment. Dysphagia. 2002;17(2):139-146.

6. White GN, O'Rourke F, Ong BS, Cordato DJ, Chan DK. Dysphagia: causes, assessment, treatment, and management. Geriatrics. 2008;63(5): 15-20.

7. Schmidt J, Holas M, Halvorson K, Reding M. Videofluoroscopic evidence of aspiration predicts pneumonia and death but not dehydration following stroke. Dysphagia. 1994;9(1):7-11.

8. Holas MA, Depippo KL, Reding MJ. Aspiration and relative risk of medical complications following stroke. Arch Neurol. 1994;51(10):1051-1053.

9. Chen PH, Golub JS, Hapner ER, Johns MM. Prevalence of perceived dysphagia and quality-of-life impairment in a geriatric population. Dysphagia. 2009;24(1):1-6. 
10. Altman KW, Yu G, Schaefer SD. Consequence of dysphagia in the hospitalized patient: impact on prognosis and hospital resources. Arch Otolaryngol Head Neck Surg. 2010;136(8):784-789.

11. Robbins J, Gangnon RE, Theis SM, Kays SA, Hewitt AL, Hind JA. The effects of lingual exercise on swallowing in older adults. J Am Geriatr Soc. 2005;53(9):1483-1489.

12. Nagai H, Russell JA, Jackson MA, Connor NP. Effect of aging on tongue protrusion forces in rats. Dysphagia. 2008;23(2):116-121.

13. Ota F, Connor NP, Konopacki R. Alterations in contractile properties of tongue muscles in old rats. Ann Otol Rhinol Laryngol. 2005;114(10): 799-803.

14. Butler SG, Stuart A, Leng X, et al. The relationship of aspiration status with tongue and handgrip strength in healthy older adults. J Gerontol A Biol Sci Med Sci. 2011;66A(4):452-458.

15. Feng X, Todd T, Lintzenich CR, et al. Aging-related geniohyoid muscle atrophy is related to aspiration status in healthy older adults. J Gerontol A Biol Sci Med Sci. 2013;68(7):853-860.

16. Molfenter SM, Amin MR, Branski RC, et al. Age-related changes in pharyngeal lumen size: a retrospective MRI analysis. Dysphagia. 2015; 30(3):321-327.

17. Aminpour S, Leonard R, Fuller SC, Belafsky PC. Pharyngeal wall differences between normal younger and older adults. Ear, Nose ThroatJ. 2011;90(4):E1-E5.

18. Langmore SE, Pisegna JM. Efficacy of exercises to rehabilitate dysphagia: a critique of the literature. Int J Speech Lang Pathol. 2015;17(3): 222-229.

19. Rosenbek JC, Robbins JA, Roecker EB, Coyle JL, Wood JL. A penetration-aspiration scale. Dysphagia. 1996;11(2):93-98.

20. Martin-Harris B, Logemann JA, McMahon S, Schleicher M, Sandidge J. Clinical utility of the modified barium swallow. Dysphagia. 2000; 15(3):136-141.

21. Hazelwood RJ, Armeson KE, Hill EG, Bonilha HS, Martin-Harris B. Identification of swallowing tasks from a modified barium swallow study that optimize the detection of physiological impairment. J Speech Lang Hear Res. 2017;60(7):1855-1863.

22. Robbins J, Butler SG, Daniels SK, et al. Swallowing and dysphagia rehabilitation: translating principles of neural plasticity into clinically oriented evidence. J Speech Lang Hear Res. 2008;51(1):S276.

23. Pisegna JM, Kaneoka A, Pearson WG, Kumar S, Langmore SE. Effects of non-invasive brain stimulation on post-stroke dysphagia: a systematic review and meta-analysis of randomized controlled trials. Clin Neurophysiol. 2016;127(1):956-968.

24. Burkhead LM, Sapienza CM, Rosenbek JC. Strength-training exercise in dysphagia rehabilitation: principles, procedures, and directions for future research. Dysphagia. 2007;22(3):251-265.

25. Lazarus C, Logemann JA, Gibbons P. Effects of maneuvers on swallowing function in a dysphagic oral cancer patient. Head Neck. 1993;15(5):419-424.
26. Hammer MJ, Jones CA, Mielens JD, Kim CH, McCulloch TM. Evaluating the tongue-hold maneuver using high-resolution manometry and electromyography. Dysphagia. 2014;29(5):564-570.

27. Martin BJ, Logemann JA, Shaker R, Dodds WJ. Normal laryngeal valving patterns during three breath-hold maneuvers: a pilot investigation. Dysphagia. 1993;8(1):11-20.

28. Shaker R, Kern M, Bardan E, et al. Augmentation of deglutitive upper esophageal sphincter opening in the elderly by exercise. Am J Physiol. 1997;272(6 Pt 1):G1518-G1522.

29. Kahrilas PJ, Logemann JA, Krugler C, Flanagan E. Volitional augmentation of upper esophageal sphincter opening during swallowing. Am J Physiol. 1991;260(3 Pt 1):G450-G456.

30. Miloro KV, Pearson WG, Langmore SE. Effortful pitch glide: a potential new exercise evaluated by dynamic MRI. $J$ Speech Lang Hear Res. 2014;57(4):1243-1250.

31. Martin-Harris B, Brodsky MB, Michel Y, et al. MBS measurement tool for swallow impairment-MBSImp: establishing a standard. Dysphagia. 2008;23(4):392-405.

32. Steele CM, Grace-Martin K. Reflections on clinical and statistical use of the Penetration-Aspiration scale. Dysphagia. 2017;32(5):601-616.

33. Robbins J. Guest editorial: the current state of clinical geriatric dysphagia research. Journal of Rehabilitation Research and Development. 2002;39(4):ix

34. Roy N, Stemple J, Merrill RM, Thomas L. Dysphagia in the elderly: preliminary evidence of prevalence, risk factors, and socioemotional effects. Ann Otol Rhinol Laryngol. 2007;116(11):858-865.

35. Bloem BR, Lagaay AM, van Beek W, Haan J, Roos RA, Wintzen AR. Prevalence of subjective dysphagia in community residents aged over 87. BMJ. 1990;300(6726):721-722.

36. Yang EJ, Kim MH, Lim JY, Paik NJ. Oropharyngeal dysphagia in a community-based elderly cohort: the Korean longitudinal study on health and aging. J Korean Med Sci. 2013;28(10):1534-1539.

37. Kikawada M, Iwamoto T, Takasaki M. Aspiration and infection in the elderly. Drugs Aging. 2005;22(2):115-130.

38. Stephen JR, Taves DH, Smith RC, Martin RE. Bolus location at the initiation of the pharyngeal stage of swallowing in healthy older adults. Dysphagia. 2005;20(4):266-272.

39. Martin-Harris B, Brodsky MB, Michel Y, Lee FS, Walters B. Delayed initiation of the pharyngeal swallow: normal variability in adult swallows. J Speech Lang Hear Res. 2007;50(3):585-594.

40. Pearson WG, Hindson DF, Langmore SE, Zumwalt AC. Evaluating swallowing muscles essential for hyolaryngeal elevation by using muscle functional magnetic resonance imaging. Int $J$ Radiat Oncol Biol Phys. 2013;85(3):735-740.
Clinical Interventions in Aging

\section{Publish your work in this journal}

Clinical Interventions in Aging is an international, peer-reviewed journal focusing on evidence-based reports on the value or lack thereof of treatments intended to prevent or delay the onset of maladaptive correlates of aging in human beings. This journal is indexed on PubMed Central, MedLine,
Dovepress

CAS, Scopus and the Elsevier Bibliographic databases. The manuscript management system is completely online and includes a very quick and fair peer-review system, which is all easy to use. Visit http://www.dovepress. com/testimonials.php to read real quotes from published authors. 